\title{
L. GuMAŃSKI
}

\section{REMARKS ON FORMALIZED PROOF AND CONSEQUENCE}

\author{
(Summary)
}

The familiar definition of "proof" (cf. (D1); the symbol " $D w_{R}[W, Z]$ " is to be read "proof cf the expression $W$ on the grourds cf the assumptions which constitute the set $Z$ and rules of inference which constitute the set $R$ "; the symbol

$$
r \frac{U_{J}, \ldots, U_{j_{m}}}{W_{i}^{\prime}}
$$

is to be read "rule of inference $r$ permits to allow $W_{i}$ on the grounds of $U_{j_{1}}, \ldots, U_{j_{m}}$ ") is unsatisfactory because a proof in this sense $1^{\circ}$ can contain - as A. Church mentioned - inessential elements, and $2^{\circ}$ it can perpetrate a circulus vitiosus in demonstrardo. Hence other definitions of "proof" are considered ((D2)-(D6); " $\phi$ " is to be read "is a proper subsequence of the sequence", " $m$ " is to be read "is an element of the sequence"). Among these definitions (D4) seems to be closest to the semantic intuitions which we usually link to the expression "(formalized) proof which is gocd formally". Then the set of axioms (A1)-(A7) of the notion of $R$-consequence of the set $X\left(C n_{R}(X)\right)$ is given. The set (A1)-(A7) is adapt:d to (D3) in a way analogous to that in which Tarski's sct of axioms (1)-(4) is adaptcd to (D1). The axioms (A1)-(A7) which are in a sense equivalent to the axioms (1)-(4) employ to the discussion of matters connected with the notion of consequence the (primitive) terms closer to current intuitions than Tarski's primitive terms. 